\title{
Colgajo en alas: una alternativa para la reconstrucción de las neosindactilias cicatriciales postquemaduras pediátricas*
}

\author{
Drs. RICARDO AYALA M. ${ }^{1}$, MARÍA BEATRIZ QUEZADA K. ${ }^{1}$, \\ CECILIA BRIONES S. ${ }^{1}$, FRESIA SOLÍS F. ${ }^{2}$
}

\begin{abstract}
Centro de Rehabilitación Santiago de la Corporación de Ayuda al Niño Quemado, COANIQUEM.
2 Dirección de docencia, investigación y extensión de la Corporación de Ayuda al Niño Quemado, COANIQUEM. Santiago, Chile.
\end{abstract}

\begin{abstract}
\section{Use of wing flaps to correct web space sequels of hand burns in children}

Background: With finger growth, sequels of burns in children located in the palm of the hand and web spaces tend to form syndactylyes, which can limit the opening of fingers. These sequels need a reconstructive surgical approach. Aim: To describe the use of flap wings for syndactylyes occurring after burns in children and analyze its surgical results. Materials and methods: Retrospective review of 50 medical records of children undergoing surgery using the flap wings technique in the hand, collecting demographic, clinical and surgical variables. Results: In the 50 children analyzed, 69 surgical procedures using the flap wing technique were performed, corresponding to 1.38 procedures per patient. The burn occurred at $2.2 \pm 3.0$ years of age. The surgical procedures were carried out $6.7 \pm 4.5$ years after the burn episode, at $8.9 \pm 4.9$ years of age. Wing flaps were located predominantly in second and third web spaces. Average operating time was $72.2 \pm 34$ minutes. Only three (6\%) children had minor complications. Conclusions: The proposed surgical technique is a useful surgical approach for the burned web space of the hand, with a low proportion of complications.

Key words: Burns, hand, flap wing.
\end{abstract}

\section{Resumen}

Introducción: Con el crecimiento de los dedos, las secuelas postquemaduras infantiles localizadas a nivel de la palma y comisuras tenderán a formar neosindactilias cicatriciales, lo que puede limitar la apertura de los dedos, necesitando un abordaje quirúrgico reconstructivo. Objetivo: Describir la técnica "colgajo en alas" en las neosindactilias cicatriciales post quemaduras en niños y, analizar los resultados de las cirugías en que se ha utilizado este procedimiento. Materiales y Métodos: Estudio descriptivo retrospectivo, de revisión aleatoria de 50 fichas de niños sometidos a cirugía con colgajo en alas en mano, de un total de 980 cirugías de mano realizadas en COANIQUEM por cualquier técnica, entre los años 2000-2009, recolectando variables demográficas, clínicas y quirúrgicas. Resultados: Se realizan $69 / 50=1,38$ cirugías por paciente

* Recibido el 23 de mayo de 2012 y aceptado para publicación el 29 de junio de 2012.

Los autores no refieren conflictos de interés.

Correspondencia: Dra. María Beatriz Quezada K.

San Francisco 8586, Pudahuel, Santiago, Chile.

beatrizquezadakerr@yahoo.com 
usando la técnica de colgajo en alas. La quemadura se produjo en promedio a los 2,2 $\pm 3,0$ años; la cirugía se realizó a los 6,7 $\pm 4,5$ años de evolución y a los 8,9 $\pm 4,9$ años de edad. El tiempo de cirugía en promedio duró $72,2 \pm 34,7$ minutos; los colgajos en alas se localizaron con mayor frecuencia en segunda y tercera comisura. Sólo el $6 \%$ de los niños presentó complicaciones menores (3/50). Conclusiones: La técnica quirúrgica propuesta, constituye una herramienta útil en el abordaje de las neosindactilias cicatriciales postquemaduras, de gran versatilidad de uso hacia palmar, dorsal o comisuras contiguas y baja proporción de complicaciones.

Palabras clave: Neosindactilia, técnica de colgajo en alas, secuela de quemadura.

\section{Introducción}

Las secuelas de quemadura de mano en los niños son complejas. La lesión ocurre en la mayoría de los casos entre los 2 y 5 años de vida, implicando que la rehabilitación se ve dificultada por el pequeño tamaño de la mano y la escasa colaboración del niño a esa edad. Además el crecimiento, especialmente en aquellas secuelas que incluyen varios dedos y la palma, provocará un rápido compromiso funcional de la mano que requerirá múltiples cirugías para su tratamiento ${ }^{1}$.

Los niños poseen características físicas propias de la edad, mayor cantidad de tejido adiposo, especialmente en dorso, por lo que es menos probable la exposición de tendones, articulaciones etc. tanto en el período agudo como en la cirugía reconstructiva. La piel de los niños es más elástica y más fina, lo que permite mayor facilidad para desplazar y avanzar colgajos. También poseen mayor tendencia a la cicatrización hipertrófica postquemaduras y, a la retracción con formación de bridas que cruzan varias zonas articulares ${ }^{2,3}$.

Con el crecimiento de los dedos, aquellas secuelas localizadas a nivel de la palma, dorso y comisuras tenderán a formar neosindactilias cicatriciales que darán un aspecto característico de "aleta de pato". A pesar que la mayoría de las veces el compromiso es sólo estético, puede limitar la apertura de los dedos, especialmente cuando se localiza a nivel de primera y segunda comisura.

Existen múltiples técnicas quirúrgicas para la resolución de las neosindactilias, como: zetoplastías (simples, dobles o de cuatro colgajos), colgajos de transposición, avance y rotación ${ }^{4-11}$ (Figura 1).

En la Corporación de Ayuda al Niño Quemado (COANIQUEM), entre los años 2000 y 2009, se realizaron 980 cirugías en manos usando diferentes técnicas, tanto en fase aguda como de secuela; estas últimas, correspondían al 45,9\%. El modelo de cirugía ambulatoria en la institución, considera al niño como ser integral, otorgándole preparación emocional para enfrentar la intervención, de tal manera que no sea una experiencia traumática; además, le permite que participe en la elección del mejor momento para realizar la cirugía y colabore más activamente en su rehabilitación.

El objetivo de este trabajo es describir la técnica que hemos denominado "colgajo en alas" en las neosindactilias cicatriciales post quemaduras en niños y analizar los resultados de las cirugías en que se ha utilizado esta técnica.

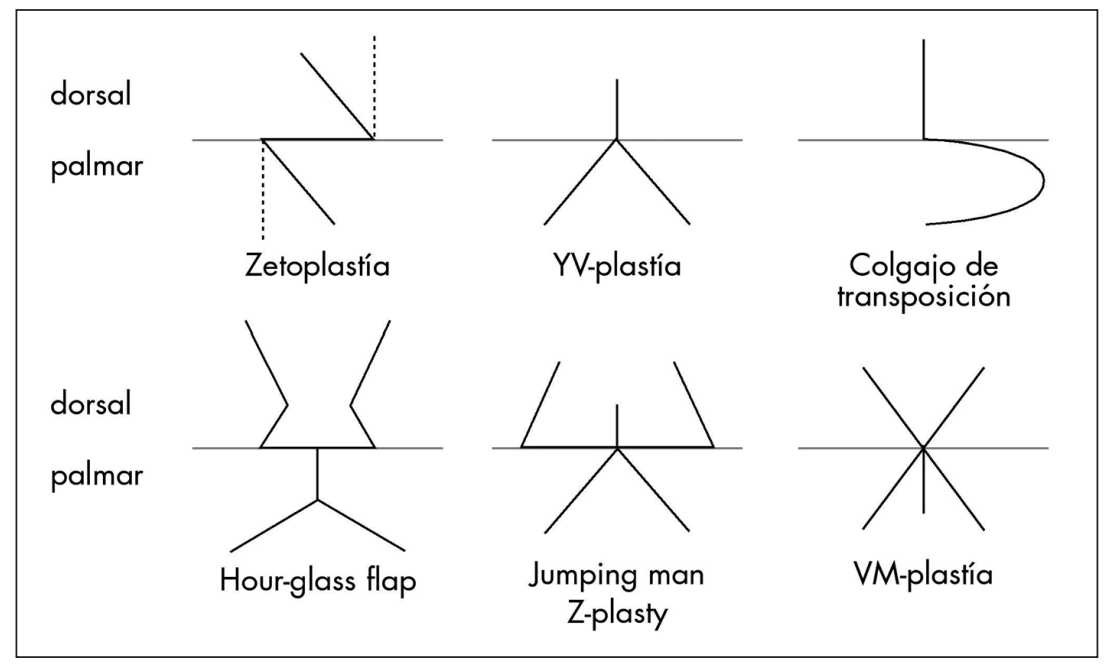

Figura 1. Técnicas quirúrgicas de resolución de neosindactilias. 


\section{Materiales y Métodos}

Estudio descriptivo retrospectivo, de revisión aleatoria simple de 50 fichas de niños sometidos a cirugía con colgajo en alas $(11,1 \%$ del total), realizadas en COANIQUEM, entre los años 2000-2009.

De la ficha, se recolectan las siguientes variables demográficas y clínicas: género, edad del niño al momento de la quemadura, tiempo de la secuela, edad del niño al momento de la cirugía, agente que produce la quemadura, localización, superficie corporal quemada, manejo y tratamiento en la etapa aguda. Variables quirúrgicas: número de alas, número de comisuras intervenidas, tiempo de proceso quirúrgico (días), tiempo de anestesia (min), tiempo operatorio ( $\mathrm{min}$ ), número de cirugías previas, lateralidad, localización de secuelas y presencia de complicaciones. Cada paciente tiene registro fotográfico en el momento de la cirugía.

Los datos se vaciaron en planilla Excel y se procesaron con SPSS versión 16.0, obteniéndose medidas de resumen de las variables indicadas.

\section{Descripción de la técnica quirúrgica}

El colgajo en alas, está diseñado para solucionar las neosindactilias cicatriciales localizadas en todas las comisuras, puede ser utilizado hacia palmar o hacia dorsal; consiste en levantar 2 colgajos alargados de las caras cubital y radial de dedos adyacentes con cuidado de no lesionar los paquetes vasculonerviosos. Se diseca la piel que constituye la neosindactilia en sentido proximal hasta lograr visualizar tejido celular sin fibrosis a nivel de la comisura. Se incinde la piel palmar en sentido vertical hasta donde la parte central del colgajo se pueda avanzar y luego se incinde en sentido horizontal a modo de $T$ invertida seccionando la cicatriz retráctil generándose una zona cruenta que es ocupada por la rotación de los colgajos en alas. La piel palmar se avanza hacia la cara lateral de los dedos sin tensión. Se sutura la piel con monofilamento 5,0 continuo. Esta técnica se realiza con isquemia, la cual se termina una vez que se efectúa el vendaje, dejando las falanges distales expuestas para evaluar irrigación (Figura 2).

\section{Resultados}

De los 50 pacientes, el $62 \%$ correspondió al género masculino; la quemadura se produjo en promedio a los 2,2 $\pm 3,0$ años de edad; se realizó rehabilitación de la secuela por un período de $6,7 \pm 4,5$ años y la cirugía reconstructiva se realizó a los $8,9 \pm 4,9$ años de edad. El objeto caliente se constituyó en el principal agente causal (48\%) de la quemadura. En el $84 \%$ de los casos, el manejo de niños en etapa aguda se realizó en forma externa a COANIQUEM. El $86 \%$ de los niños fue tratado con injertos dermoepidérmicos (Tabla 1).

Se realizan $69 / 50=1,38$ cirugías por paciente usando la técnica de colgajo en alas. Cuando se efectúa un colgajo en alas, el 36,4\% correspondió a la segunda comisura. Si se realizan dos colgajos, el $83,3 \%$ se localiza en segunda y tercera comisura $y$, al realizar tres colgajos, 4/6 se ubica en tercera y cuarta comisura (Tabla 2).

El tiempo de proceso quirúrgico, que corresponde al período entre la fecha de operación y fecha de alta en enfermería, duró en promedio 14,84 $\pm 5,09$

Tabla 1. Características demográficas y clínicas de los pacientes

\begin{tabular}{|c|c|c|}
\hline Característica & n & $\%$ \\
\hline Total & 50 & 100 \\
\hline \multicolumn{3}{|l|}{ Género } \\
\hline Masculino & 31 & 62 \\
\hline Femenino & 19 & 38 \\
\hline \multicolumn{3}{|l|}{ Agente } \\
\hline Sólido & 24 & 48 \\
\hline Fuego & 13 & 26 \\
\hline Líquido & 11 & 22 \\
\hline Electricidad & 2 & 4 \\
\hline \multicolumn{3}{|c|}{ Manejo de niños en etapa aguda } \\
\hline Externo & 42 & 84 \\
\hline COANIQUEM & 8 & 16 \\
\hline \multicolumn{3}{|l|}{ Edad (años) } \\
\hline Edad a la quemadura & \multicolumn{2}{|c|}{$2,2 \pm 3,0$} \\
\hline Años de secuela & \multicolumn{2}{|c|}{$6,7 \pm 4,5$} \\
\hline Edad a la cirugía & \multicolumn{2}{|c|}{$8,9 \pm 4,9$} \\
\hline \multicolumn{3}{|l|}{ Localización } \\
\hline Sólo mano & 33 & 66 \\
\hline Extremidad superior & 6 & 12 \\
\hline Múltiples & 5 & 10 \\
\hline Cara-cuello & 4 & 8 \\
\hline Tórax-abdomen & 2 & 4 \\
\hline \multicolumn{3}{|c|}{ Superficie corporal quemada (\%) } \\
\hline$<0,5$ & 23 & 46 \\
\hline $0,5-0,9$ & 12 & 24 \\
\hline $1,0-4,9$ & 9 & 18 \\
\hline $5,0-19$ & 3 & 6 \\
\hline 20 o más & 3 & 6 \\
\hline \multicolumn{3}{|c|}{ Tratamiento de niños en etapa aguda } \\
\hline Injerto dermo epidérmico & 43 & 86 \\
\hline Injerto piel total & 4 & 8 \\
\hline Curación & 2 & 4 \\
\hline Injerto & 1 & 2 \\
\hline
\end{tabular}



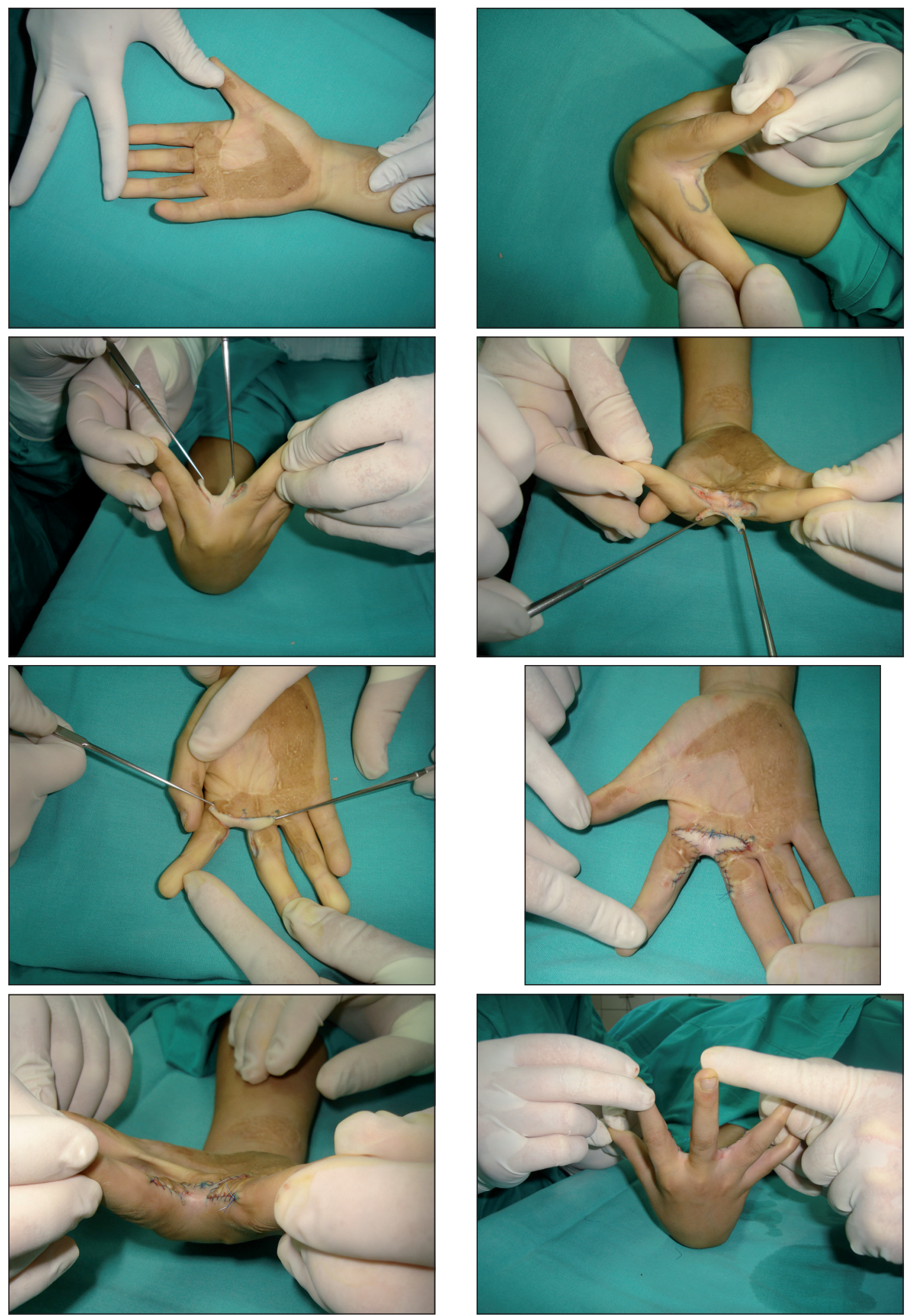

Figura 2. Técnica de colgajo en alas. 
Tabla 2. Número de alas en comisuras de la mano

\begin{tabular}{|c|c|c|c|c|c|c|c|c|c|}
\hline \multirow[t]{2}{*}{$\mathrm{n}$ alas } & \multicolumn{2}{|c|}{$1^{\mathrm{a}}$ comisura } & \multicolumn{2}{|c|}{$2^{\mathrm{a}}$ comisura } & \multicolumn{2}{|c|}{$3^{\text {a }}$ comisura } & \multicolumn{2}{|c|}{$4^{\mathrm{a}}$ comisura } & \multirow[b]{2}{*}{ Total } \\
\hline & $\mathbf{n}$ & $\%$ & $\mathbf{n}$ & $\%$ & $\mathbf{n}$ & $\%$ & $\mathbf{n}$ & $\%$ & \\
\hline 1 & 7 & 21,2 & 12 & 36,4 & 9 & 27,3 & 5 & 15,1 & 33 \\
\hline 2 & 1 & 3,3 & 13 & 43,3 & 12 & 40,0 & 4 & 13,4 & 30 \\
\hline 3 & 1 & 16,7 & 1 & 16,7 & 2 & 33,3 & 2 & 33,3 & 6 \\
\hline Total & 9 & 13,0 & 26 & 37,7 & 23 & 33,3 & 11 & 16,0 & 69 \\
\hline
\end{tabular}

Tabla 3. Estadísticas descriptivas del proceso quirúrgico

\begin{tabular}{|c|c|c|c|c|c|c|c|}
\hline Variable & \multicolumn{2}{|l|}{$\mathbf{n}$} & \multicolumn{2}{|l|}{ Mínimo } & \multicolumn{2}{|l|}{ Máximo } & $\mathbf{X} \pm \mathrm{DS}$ \\
\hline Tiempo proceso quirúrgico (días)* & \multicolumn{2}{|l|}{50} & \multicolumn{2}{|l|}{7} & \multicolumn{2}{|l|}{32} & $14,84 \pm 5,09$ \\
\hline Tiempo anestesia (min) & \multicolumn{2}{|l|}{50} & \multicolumn{2}{|l|}{45} & \multicolumn{2}{|l|}{300} & $96,86 \pm 40,26$ \\
\hline Tiempo cirugía (min) & \multicolumn{2}{|l|}{50} & \multicolumn{2}{|l|}{32} & \multicolumn{2}{|l|}{235} & $72,18 \pm 34,68$ \\
\hline $\mathrm{N}^{\mathrm{o}}$ cirugías previas & \multicolumn{2}{|l|}{50} & \multicolumn{2}{|l|}{3} & \multicolumn{2}{|l|}{11} & $7,16 \pm 2,57$ \\
\hline Presencia complicaciones & Sí = 3; & $6 \%$ & No $=47$ & $94 \%$ & & & \\
\hline Lateralidad & Izquierdo = 21; & $4,2 \%$ & Derecho $=9$ & $58 \%$ & & & \\
\hline \multirow[t]{3}{*}{ Localización secuela } & Palma $=$ & 35 & Dorso $=$ & 13 & Pulgar $=$ & 30 & Índice $=$ \\
\hline & Medio $=$ & 43 & Anular $=$ & 36 & Meñique = & 29 & $1^{\mathrm{a}}$ Comisura $=26$ \\
\hline & $2^{\mathrm{a}}$ Comisura $=$ & 42 & $3^{\mathrm{a}}$ Comisura $=$ & 35 & $4^{\mathrm{a}}$ Comisura $=$ & $=27$ & Total $=$ \\
\hline \multirow[t]{2}{*}{ Otras técnicas quirúrgicas } & Amputación $=$ & 4 & $\mathrm{D}+\mathrm{I} \mathrm{PT}=$ & 7 & $\mathrm{D}+\mathrm{IDE}=$ & 1 & \\
\hline & Colgajos simples & $=14$ & Colgajo rotaciór & $=14$ & Zetoplastía $=$ & 10 & Total $=$ \\
\hline
\end{tabular}

*Diferencia fecha operación y fecha de alta en enfermería.

días. El tiempo de la cirugía, fluctuó entre 32 y 235 minutos. Las otras técnicas empleadas en el mismo acto quirúrgico fueron zetoplastías, colgajos simples y de rotación y se ubicaron con mayor frecuencia en dedos índice y medio.

El 6\% de los niños presentó complicaciones En 3 pacientes se produjeron complicaciones catalogadas como menores porque no requieren reoperación y no retrasan el inicio de la rehabilitación. En un caso el paciente presentó una dermatitis, el segundo necrosis pequeña de punta de colgajo y el tercero, dehiscencia mínima en sutura del dedo meñique.

La rehabilitación se inició en promedio a los 11 días postoperatorio y el alta de enfermería a los 36 días (Tabla 3).

El resultado de la cirugía con la técnica de colgajo en alas a tres años de observación, se observa en Figura 3.

\section{Discusión}

En la mayoría de los pacientes, la rehabilitación nos permite integrar al niño a sus actividades de la vida diaria, que manifieste algún grado de compromiso funcional y que participe activamente en la toma de decisiones quirúrgicas.

Para decidir el mejor momento para realizar la cirugía, es necesario evaluar el compromiso funcional que la secuela provoca. Muchas veces se observan bridas o zonas de tensión que al niño no le molestan y no interfieren con sus actividades de la vida diaria. En estos casos valoramos la opinión del niño, si asegura que "no le molesta", es mejor esperar.

Las neosindactilias cicatriciales son un problema muy frecuente en las secuelas que tienen compromiso de la palma y los dedos, afortunadamente en la mayoría de los casos la piel que se encuentra en las caras laterales de ambos dedos es piel sana. Esto permite levantar los colgajos en alas, disecar la comisura hasta llegar a tejido sano e interponer los colgajos hacia la palma. En la literatura se describen múltiples técnicas especialmente descritas para neosindactilias que comprometen hasta los $2 / 3$ de la primera falange, el colgajo en alas permite solucionar neosindactilias que incluso pueden sobrepasar la articulación interfalángica proximal. 

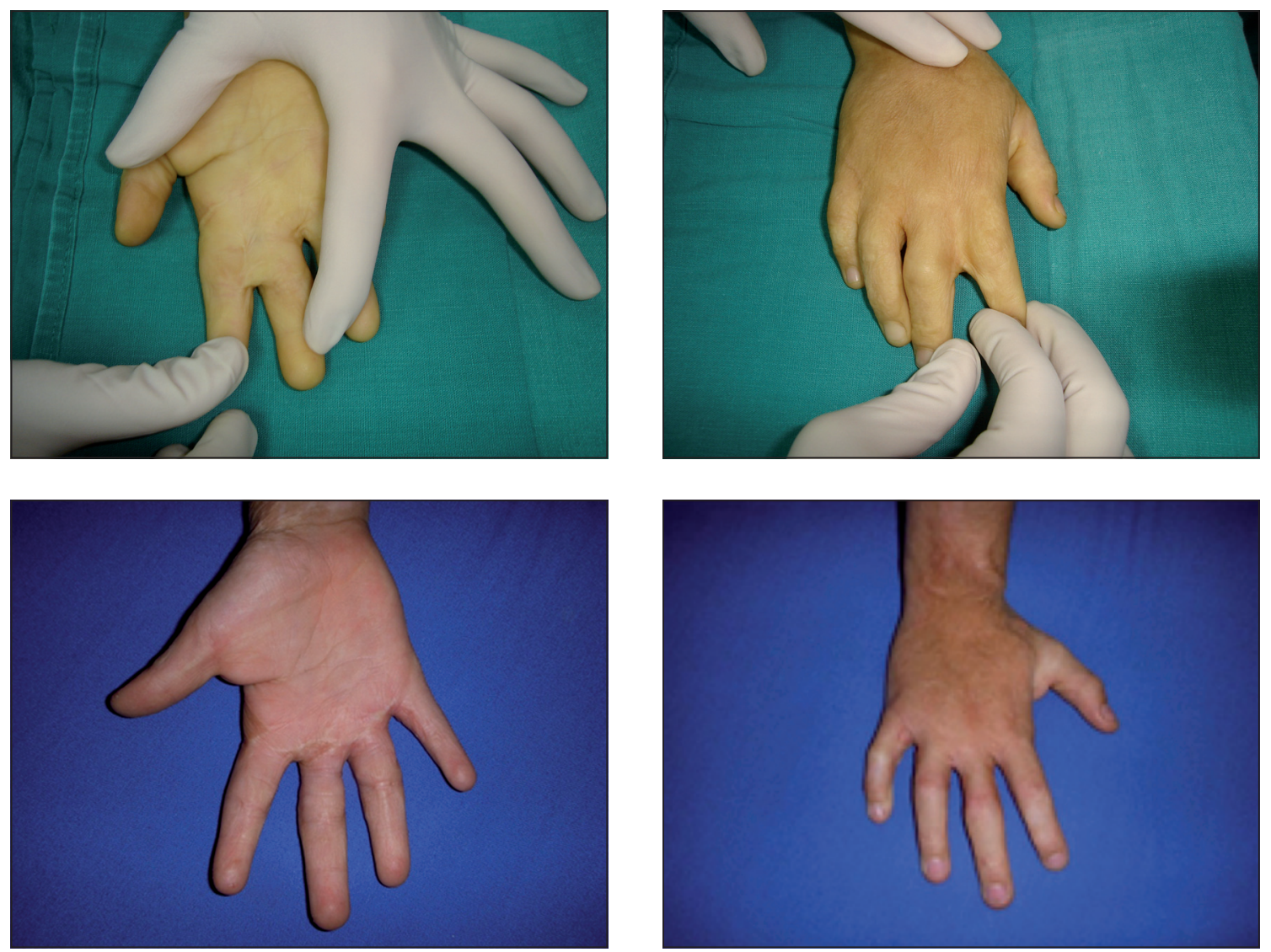

Figura 3. Resultado de la cirugía con la técnica de colgajo en alas a tres años de observación.

Además tiene la ventaja de interponer piel sana hacia la palma, solucionando no sólo la neosidactilia sino además la retracción cicatricial que impide la extensión completa de los dedos. A veces la incisión lateral en los dedos puede prolongarse hacia distal levantándose otros colgajos locales para solucionar completamente las bridas que comprometen el resto del dedo.

Otra ventaja de esta técnica es que puede utilizarse hacia palmar o hacia dorsal dependiendo de la localización del tejido cicatricial y utilizarse en todas las comisuras. Esto es posible sin problemas ya que se visualizan los paquetes vasculonerviosos.

Si faltara cobertura cutánea en la cara palmar de los dedos para poder cerrar dos comisuras contiguas, se soluciona con un injerto de piel total.

En esta técnica la colocación de los colgajos a nivel palmar, agregando piel sana con capacidad de crecer en la base de los dedos aumenta la piel de la palma, efecto aún más notorio cuando se realizan más de un colgajo en alas en comisuras contiguas.

El colgajo en alas es un procedimiento quirúrgico seguro (se presentó un mínimo de complicaciones que no retrasaron el inicio de la rehabilitación) y es una ayuda importante en cumplir el objetivo de lograr una mano completamente funcional al finalizar el crecimiento y desarrollo del niño.

\section{Referencias}

1. Robson MC, Smith Jr, Vander Zee AJ, Roberts L. Making the burned hand functional. Clin Plast Surg. 1992;19:663-71.

2. Davoodi P, Fernández JM, O SJ. Postburn sequelae in the pediatric patient: clinical presentations and treatment options. Craniofac Surg. 2008;19:1047-52.

3. Berman B, Viera MH, Amini S, Huo R, Jones IS. Prevention and management of hypertrophic scars and keloids after burns in children. J Craniofac Surg. 2008;19:989-1006.

4. Grishkevich VM. First web space post-burn contracture types: contracture elimination methods. Burns. 2011;37:338-47.

5. Gilbert DA. An overview of flaps for hand and forearm reconstruction. Clin Plast Surg . 1981;8:129-39. 
6. MacDougal B, Wray RC Jr, Weeks PM. Lateral-volar finger flap for the treatment of burn syndactyly. Plast Reconstr Surg. 1976;57:167-71.

7. Shaw DT, Li CS, Richey D. Interdigital butterfly flap in the hand (the double opposing Z-plasty). J Bone Joint Surg Am. 1973;55:1677-9.

8. Alexander JW, MacMillan BG, Martel L.Correction of postburnsyndactyly: an analysis of children with introduction of the VM plasty and postoperative pressure inserts. Plast Reconstr Surg. 1982;70:345-52.

9. Hultman CS, Teotia S, Calvert C, Thornton S, Schram
J. STARplasty for reconstruction of the burned web space: introduction of an alternative technique for the correction of dorsal neosyndactyly. Ann Plast Surg. 2005;54:281-7.

10. Tan O, Atik B, Ergen D. Versatile use of the VM-Plasty for reconstruction of the web space. Ann Plast Surg. 2005;55:623-8.

11. Emsen IM. The cross incision plasty for reconstruction of the burned web space: introduction of an alternative technique for the correction of dorsal and volar neosyndactyly. J Burn Care Res. 2008;29:378-85. 